\title{
LAS CIUDADES, EL CAMBIO CLIMÁTICO Y LA ENERGÍA INCORPORADA
}

\author{
CITIES, CLIMATE CHANGE AND EMBODIED \\ ENERGY
}

\author{
FAVIO CHUMPITAZ REQUENA \\ Universidad de Lima \\ orcid.org/0000-0001-7879-3758
}

El desarrollo de nuestras urbes es el causante del más del $70 \%$ del dióxido de carbono $\left(\mathrm{CO}_{2}\right)$ que se produce y posee un gran impacto sobre el medio ambiente. Las consecuencias de estas formas de "desarrollo" ya están presentes y se incrementarán en las siguientes décadas. La reducción del impacto depende de diversos factores, entre ellos, de la energía incorporada como una posibilidad en la arquitectura y el urbanismo, a fin de ser utilizada como una herramienta general para toda infraestructura. Bajo este criterio, la energía incorporada puede constituirse en un nuevo punto de partida que formule cuestionamientos respecto de los planteamientos arquitectónicos y urbanísticos tradicionales, a partir de nuevas prioridades materiales y sociales.

energía incorporada, ciudades, cambio climático, urbanismo, $\mathrm{CO}_{2}$
Recibido: 30 de junio del 2020

Aprobado: 18 de noviembre del 2020

doi: https://doi.org/10.26439/limaq2021.n008.5549

The development of our cities is the cause of more than $70 \%$ of the production of $\mathrm{CO}_{2}$ and has a significant impact on the environment. The consequences of these forms of "development" are already present and will increase in the following decades. The reduction of their impact depends on several factors, among them, the incorporation of energy as a possibility in architecture and urban planning, in order to be used as a general tool for all infrastructure. Under this criterion, incorporated energy can become a new starting point, formulating questions regarding traditional architectural and urban planning approaches based on new material and social priorities.

incorporated energy, cities, climate change, urbanism, $\mathrm{CO}_{2}$ 


\section{LA INTRODUCCIÓN AL ANTROPOCENO}

El cambio climático genera muchos debates en torno a su impacto, pertinencia e incluso a su existencia. Algunas personas consideran que no es un tema que debería ser abordado como urgente o primordial, debido a que el mundo se encuentra siempre en un constante cambio y lo que ocurre con el clima es parte de un ciclo que se autorregula. Sin embargo, existen vastas y diversas fuentes de información difundidas respecto a este tema que demuestran las incidencias y cambios que se vienen dando en nuestros climas, haciendo énfasis en las repercusiones que tiene sobre el planeta. Entre las principales fuentes de información están la Organización de las Naciones Unidas (ONU) y el Grupo Intergubernamental de Expertos sobre el Cambio Climático (IPCC, por sus siglas en inglés), además de cientos de organizaciones y centros de investigación de diversas universidades que siguen alertándonos y aportando información sobre esta problemática.

\section{El mundo se ha vuelto más cálido}

Temperatura media anual de la Tierra por encima o por debajo del promedio $\left({ }^{\circ} \mathrm{C}\right)$

Figura 1.

Temperatura media

anual de la Tierra

por encima o por

debajo del promedio

Fuente:

Giles y Song (2020)

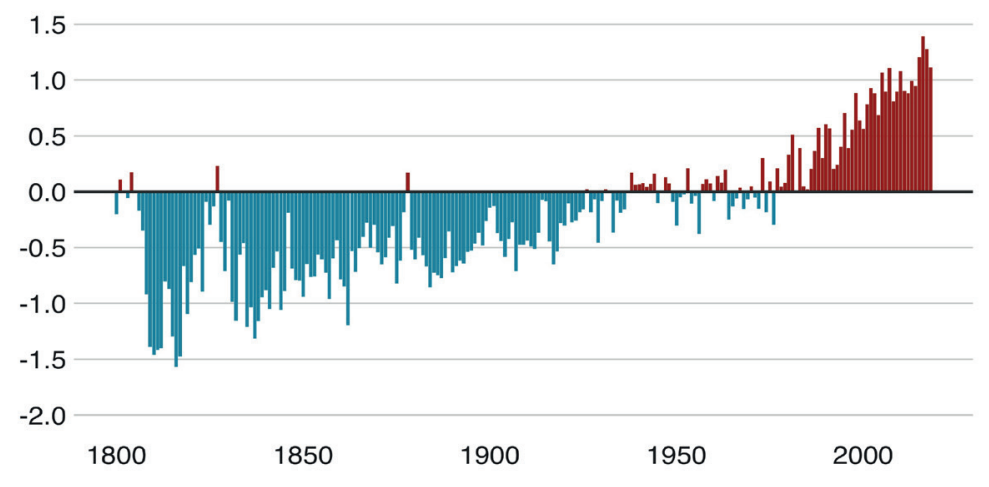

Algunas de las principales incidencias del cambio climático se pueden advertir a través del aumento sostenido de la temperatura en los últimos años. Según la Organización de las Naciones Unidas, la temperatura se incrementará en 1,5 a 2 grados para mediados del año 2050. Esto marcará un punto de no retorno en diversos ecosistemas, climas, comunidades y ciudades, que se verán considerablemente afectadas en sus formas de habitar.

Las repercusiones se darán a toda escala. Al respecto, existe un debate sobre la incidencia que genera el cambio climático en la composición geológica. Diversos especialistas en el tema, como el premio nobel de química Paul Crutzen $^{1}$, cuestionan si estamos ante una nueva era geológica, la era del Antropoceno. Esto se debe principalmente a la presencia de microplásticos

1 Paul Crutzen fue ganador del Premio Nobel de Química en 1995 junto con Mario Molina y Frank Sherwood, en reconocimiento a su trabajo sobre la protección de la capa de ozono. 
y partículas de combustibles en los estratos sedimentarios de la Tierra, que modifican su composición y comportamiento mineral. A pesar de que oficialmente esta tesis no ha sido refrendada por la Comisión Internacional de Estratigrafía ${ }^{2}$, lo planteado por Crutzen ha sido tomado como referencia por diversas personas involucradas en distintas áreas referidas al diseño. Fairs (2018) señala, como en el evento Good Design for a Bad World 2018, que este tema fue planteado y diferentes personas mostraron sus puntos de vista y respuestas ante los efectos del cambio climático en el ambiente.

Si bien la reducción del impacto depende de diversos factores, entre los que se encuentra principalmente la generación de electricidad, transporte, industria, agricultura, ganadería y hogar; es claro que se requieren acciones globales de todos los frentes para atenuar esta crisis, o nuestras formas de convivir se verán dramáticamente afectadas. En ese sentido, es relevante, pertinente y urgente que todos los actores involucrados en la toma de decisiones de las ciudades puedan hacer seguimiento, desde lo macro a lo micro, respecto a los requerimientos y constantes cambios que van experimentando las ciudades a lo largo del tiempo. Y, sobre todo, es necesario tener especial consideración respecto de aquellas menos preparadas para asumir el impacto y las repercusiones del cambio climático, como la gran mayoría de ciudades del Perú, entre ellas, la capital, Lima.

\section{LAS CIUDADES EN EL CAMBIO CLIMÁTICO}

Debido a que las ciudades son las principales generadoras de su propio destino,

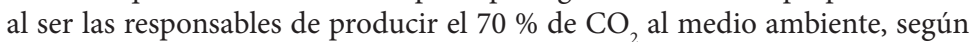
cifras del grupo $\mathrm{C}_{4} 0^{3}$, es válido cuestionarnos respecto a cómo la planificación de nuestras ciudades debe incorporar de manera más protagónica esta urgencia a fin de generar soluciones masivas que ayuden a prevenir y prepararlas para las inclemencias del cambio climático, teniendo en cuenta la gran cantidad de metros cuadrados que se construye anualmente.

Para el año 2060, el equipo liderado por el ingeniero Yet-Ming Chiang ${ }^{4}$ del MIT prevé que se duplicará en el mundo la cantidad de edificios construidos hasta el 2019, lo que equivaldrá a la construcción de una ciudad de Nueva York por mes (Sabina, 2019).

Si esta gran cantidad de volumen se ha de construir sobre el planeta en los próximos cuarenta años, es necesario poner atención a las sugerencias de los especialistas que enfocan sus estudios y propuestas en cómo estos nuevos metros cuadrados deben incluirse en la planificación de nuestras ciudades,

2 La Comisión Internacional de Estratigrafía (International Commission on Stratigraphy, ICS) es un organismo científico de la Unión Internacional de Ciencias Geológicas, que tiene dentro de sus objetivos definir la escala de tiempo geológica internacional.

3 C40 es una red de megaciudades del mundo comprometidas con abordar el cambio climático.

4 Yet-Ming Chiang es profesor de Ciencias e Ingeniería de Materiales en el Massachusetts Institute of Technology. 


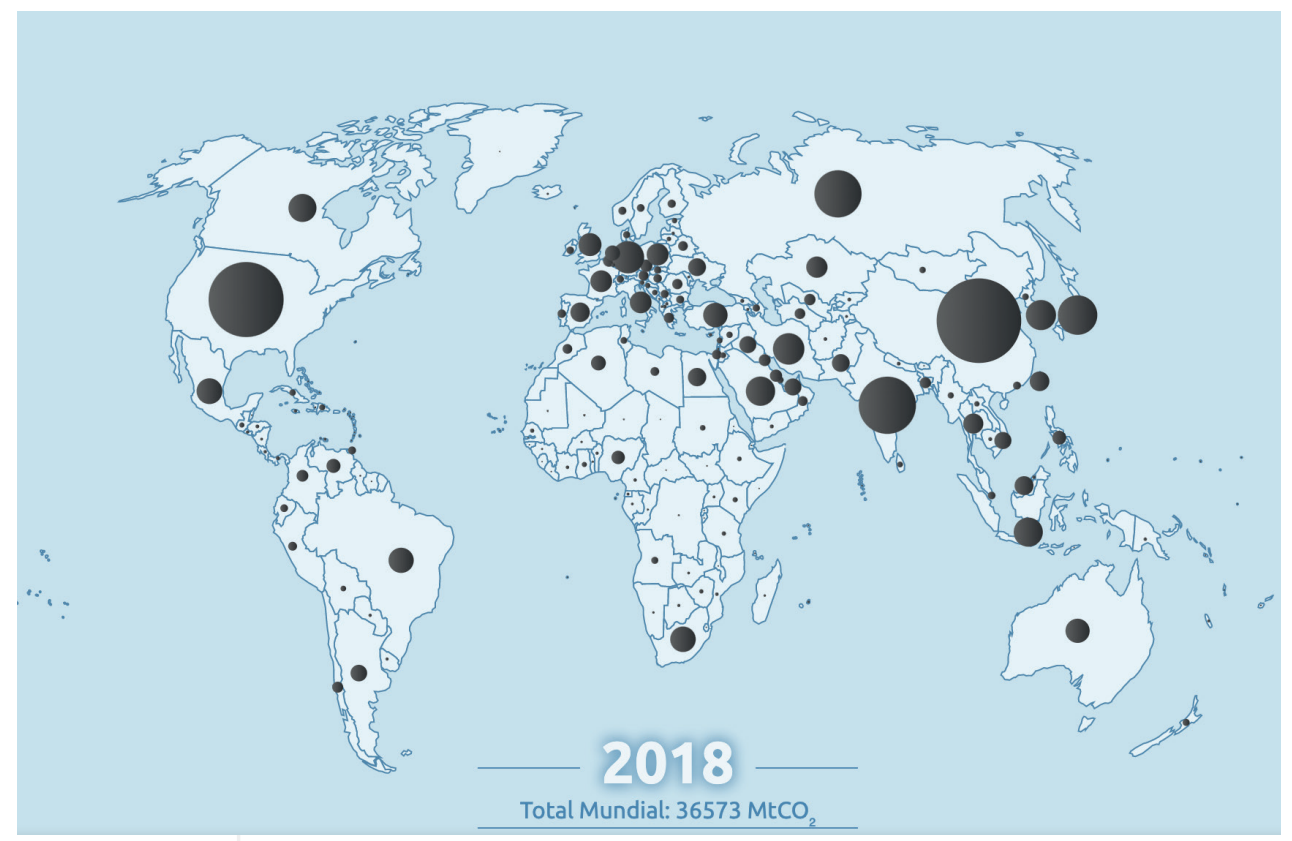

Figura 2 .

Mapamundi que indica los países con mayor generación de $\mathrm{CO}_{2}$

Nota.

El tamaño del círculo indica la proporción de $\mathrm{CO}_{2}$ que se liberó al ambiente en el 2018 (36 573

MtCO2).

Fuente:

Friedlingstein et al.

(2020) buscando urbes más sostenibles y que a su vez disminuyan el impacto del cambio climático en ellas.

Si bien varias de las ciudades en el Perú no cuentan con planes de desarrollo a futuro, algunas sí los poseen, aunque no necesariamente los ejecutan; mientras que en otros casos se vienen actualizando o desarrollando, como sucede en Lima. A nuestro parecer, esta es una oportunidad para poner mayor énfasis en la relación del cambio climático con el desarrollo futuro de las urbes.

En tal sentido, si bien existe una gran cantidad de acciones por realizar, podemos concentrar nuestros esfuerzos en tres principales propuestas que tienen más incidencia en la reducción del $\mathrm{CO}_{2}$ generado por las ciudades. Estas se encuentran referidas al uso eficiente de nuestras ciudades existentes, la concentración de futuros metros cuadrados por construir y la disminución de la huella de carbono desde diversas escalas. Todas estas propuestas tienen en común y como punto transversal la energía incorporada.

\section{I (RE)USO DE EDIFICACIONES}

Uno de los principales puntos para evitar mayores emisiones de $\mathrm{CO}_{2}$ es poder disminuir la cantidad de metros cuadrados construidos y, a su vez, hacerlos más eficientes y versátiles a futuro. En tal sentido, la reutilización, refacción y reacondicionamiento de edificaciones o zonas en desuso en las ciudades es parte de la solución. 
En los últimos años, diversos esfuerzos se realizan alrededor del mundo, poniendo este punto sobre la mesa. En el 2019, Phineas Harper, crítico y curador de diversas manifestaciones entre la arquitectura y la política, abordó el tema de la reutilización de las edificaciones como una opción de respuestas para las ciudades. El referido crítico planteó que las refacciones en las ciudades podrían asemejarse a la técnica japonesa del boro, la cual repara sus prendas a perpetuidad. Esta es una oportunidad para pensar en las edificaciones como elementos que cambian su uso o sus condiciones físicas de acuerdo con sus necesidades en el tiempo. Esto contribuiría a la reducción de las emisiones de $\mathrm{CO}_{2}$ al evitar demoliciones completas y la construcción de edificaciones desde cero.

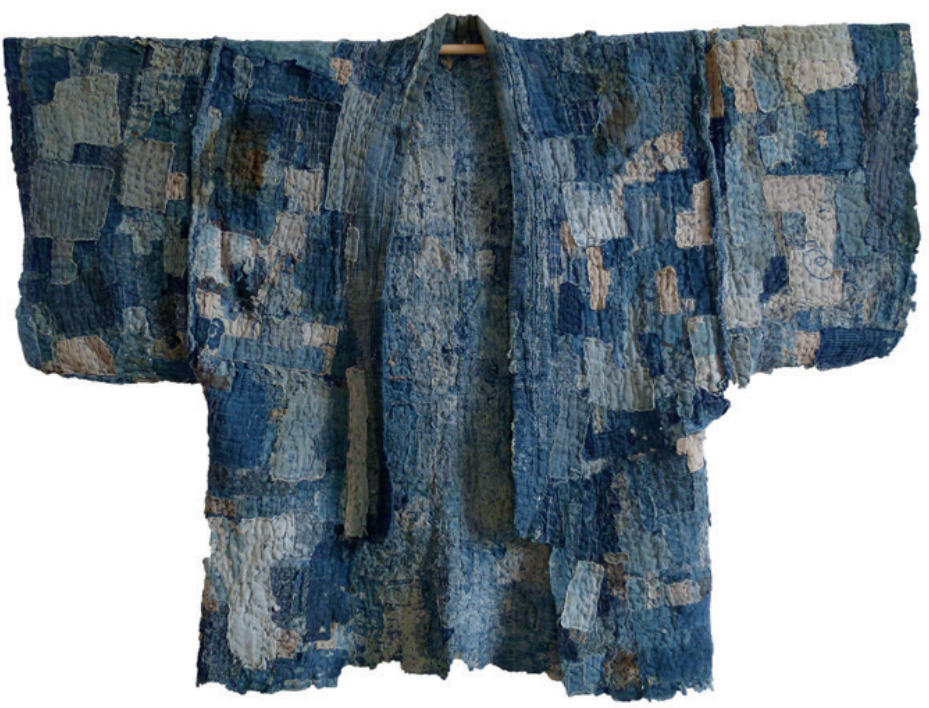

Figura 3.

Kimono tradicional de estilo boro

Fotografía: Gerrie Congdon (2020)

¿Acaso no es posible imaginar que este concepto se puede afianzar e incluir dentro de los diversos planes urbanos por desarrollar? Aplicándolo en todas las edificaciones vacías, abandonadas, en desuso (normalmente congregadas en el centro de la ciudad), o con usos ya no compatibles (antiguas zonas industriales, como la del eje de la avenida Argentina), dentro de la ciudad de Lima. Sería esta una de las principales acciones para hacer frente al cambio climático, reacomodando lo existente y anticipándonos al crecimiento de la ciudad. Esta acción debe venir acompañada con incentivos, como se brindan ya en Lima para otro tipo de edificaciones sostenibles, a las que se permite una mayor cantidad de área techada, altura en la edificación, reducción del área mínima por unidad de vivienda y menor cantidad de estacionamientos (Ordenanzas 510/MM y 539/MM).

De igual manera, potenciar los reconocimientos arquitectónicos para las edificaciones más sostenibles a raíz de una remodelación puede generar un incentivo en sus diseñadores para implementar respuestas que reduzcan el 
$\mathrm{CO}_{2}$ en sus propuestas e impulsen la generación de más variedad en los tipos de arquitectura en la ciudad, basados en este criterio.

Es importante que las ciudades puedan reutilizar al máximo las edificaciones existentes y diseñar las nuevas permitiendo una mayor flexibilidad para diversos factores funcionales, que anticipen y se adecúen a su entorno, y que puedan responder paulatinamente a los factores climáticos. De igual manera, es relevante pensar dónde se ubicarán los otros nuevos metros cuadrados que no puedan contemplarse en esta propuesta. Se trata de concentrarlos en algunas zonas de la ciudad donde sean más eficientes en el uso de energía, disminuyendo la producción de $\mathrm{CO}_{2}$.

\section{DENSIDAD EN TORNO A LA MOVILIDAD}

La conformación y crecimiento que han tenido la mayoría de nuestras ciudades las ha llevado a poseer una baja densidad, pues se han extendido de manera dispersa y en baja altura, lo que ocasiona un mayor consumo de energía en su funcionamiento y desarrollo.

Lima cuenta con una densidad de 120 hab./ha, según las cifras del Instituto Nacional de Estadística e Informática (INEI) en el 2017, lo que implica que puede y debe absorber una mayor cantidad de densidad en ella, incluso hasta duplicarla o triplicarla en distintas zonas de la ciudad. Una buena respuesta para la ciudad de Lima, que espera albergar a cinco millones de personas adicionales hasta el 2050, es disminuir el crecimiento horizontal y promover la densificación en puntos conectados de la ciudad que requerirán de viviendas y servicios.

Figura 4

Altura y densidad

en Lima

Metropolitana

Fotografía:

Andina/Carlos

Lezama (2017)

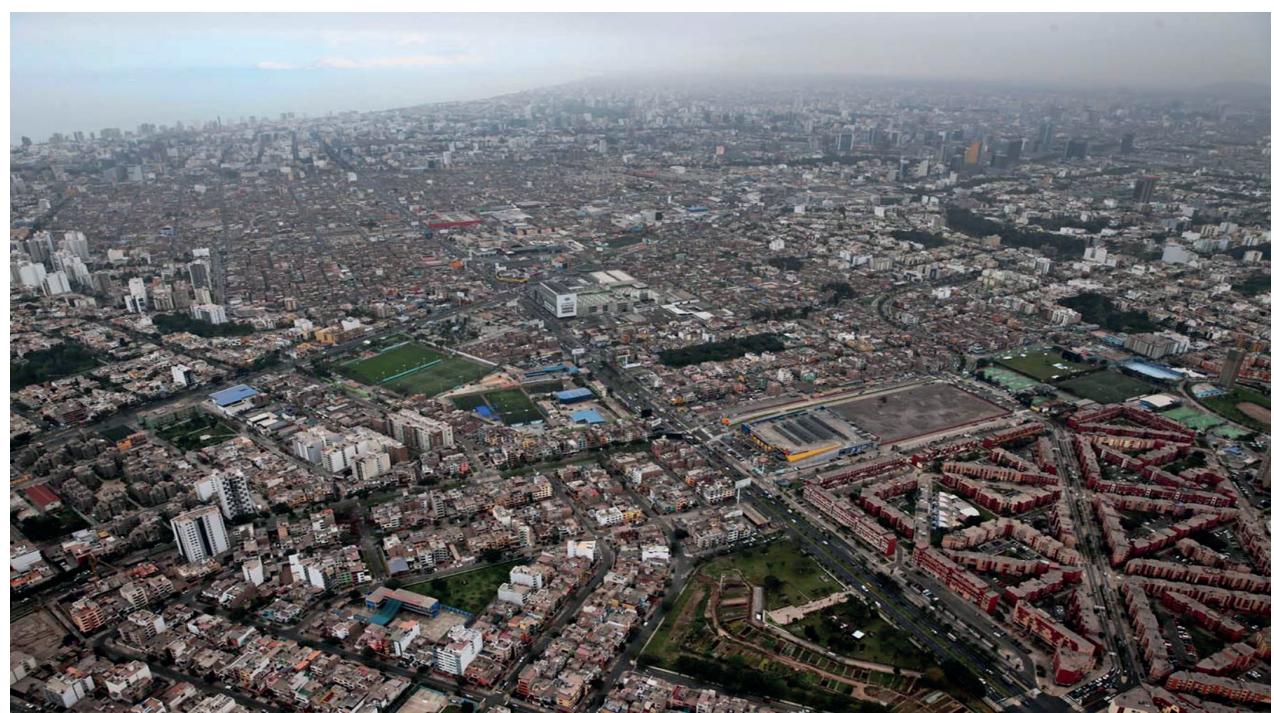


La densificación es en sí el segundo criterio importante como medida para reducir las incidencias del cambio climático. Permite poder concentrar de manera más eficiente una suma considerable de metros cuadrados, que serán añadidos a la ciudad.

Diversos especialistas coinciden en que la densificación debe concentrarse y reordenarse en torno a la relación con la movilidad de transporte masivo. Uno de ellos es Vishaan Chakrabarti ${ }^{5}$, autor del libro A Country of Cities (2013), en el que plantea su postura de la relación entre la densificación y la movilidad como aliados importantes en la búsqueda de generar ciudades sostenibles, a partir de una correlación entre nodos de movilidad del transporte masivo con la densidad de edificaciones alrededor de ellas. Su estudio plantea una densidad de 74 viviendas por hectárea (30 viviendas por acre) como la mínima eficiente para estar cerca de las zonas de transporte masivo y que produce una cantidad anual reducida de emisiones de carbono. Si trasladamos este cálculo a densidad por habitante, considerando que en varias reglamentaciones de Lima se toma como cinco habitantes máximos por unidad de vivienda de tres dormitorios, la propuesta estaría alrededor de 370 hab./ha, cerca del triple de lo que posee actualmente la capital.

Plantear esta densidad ideal, que describe el autor en una ciudad como Lima, permitiría tener una densidad idónea para el crecimiento de la ciudad en los próximos años, contribuyendo a la reducción en la producción de $\mathrm{CO}_{2}$. De esta manera, se posibilitaría una mayor eficiencia en el desplazamiento de las personas a distintas zonas de la ciudad, acompañado de micromovilidades sostenibles que los conecten en zonas cercanas.

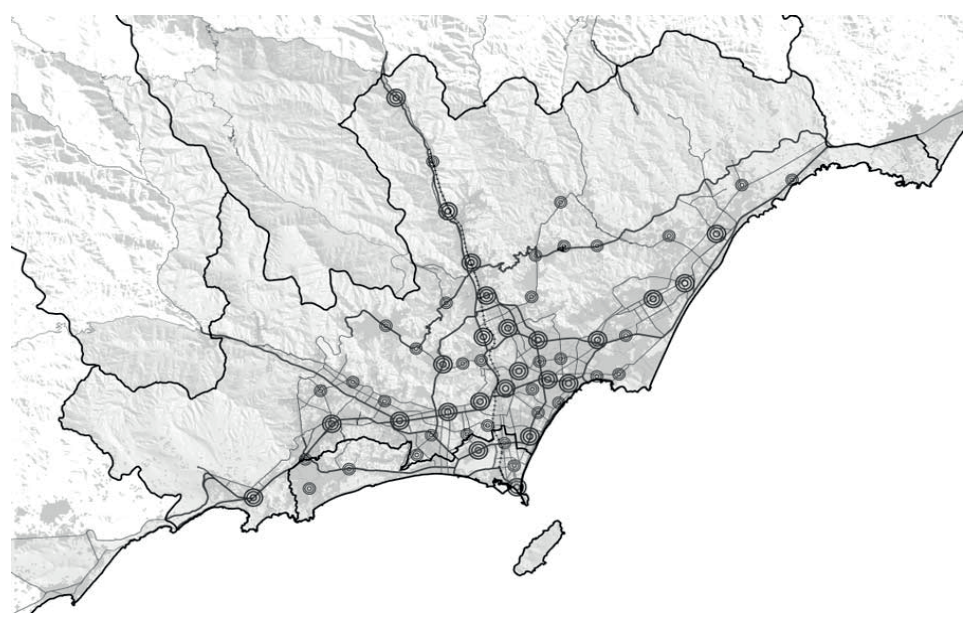

5 Vishaan Chakrabarti tiene un máster en Planificación Urbana por el Massachusetts Institute of Technology, una maestría en Arquitectura por la University of California at Berkeley y es bachiller con doble mención en Art History and Engineering por la Cornell University. 
La gran mayoría de planes de desarrollo que ha tenido Lima a lo largo del tiempo incide en generar nuevas centralidades, que en gran parte se encuentran conectadas al sistema de transporte público masivo. Estos son los lugares claves para densificar la ciudad.

Aun implementando estas dos acciones (reúso de edificaciones y densidad en torno a la movilidad a nivel macro), es relevante que cada proyecto y metro cuadrado nuevo que aparezca pueda reflexionar en su ADN reevaluando en su diseño la energía incorporada, como punto importante en su desarrollo y planificación.

\section{LA ENERGÍA INCORPORADA Y EL TIEMPO DE RETORNO}

La energía incorporada es la consumida por todos los procesos asociados a la producción de un edificio, desde la extracción de la materia prima para conformar un producto, traslado de los materiales, implementación en la edificación, hasta proponer qué pasará con ellos después de su vida útil. Priorizar la energía puede ser clave por el potencial que tiene para la disminución de la producción de $\mathrm{CO}_{2}$ al medio ambiente. Esta condición generaría nuevas lógicas conceptuales en propuestas arquitectónicas, mostrándonos edificaciones que respondan a la energía incorporada de los elementos que las componen, como punto de partida.

Las nuevas partes de las ciudades deben aprovechar el avance de la tecnología a su favor, buscando que aquellas que se incorporen no solo intenten reducir su consumo energético, sino que sean capaces de considerar en su propuesta materiales y diseños que sean adecuados para disminuir, producir o superar la energía requerida para su funcionamiento.

Powerhouse Brattørkaia es un edificio de oficinas de 18000 metros cuadrados, ubicado en Trondheim, Noruega, que constituye un claro ejemplo, puesto que dicha infraestructura produce más del doble de energía de la que consume y deriva el exceso de esta a edificios vecinos, autobuses eléctricos, automóviles y barcos de la zona.

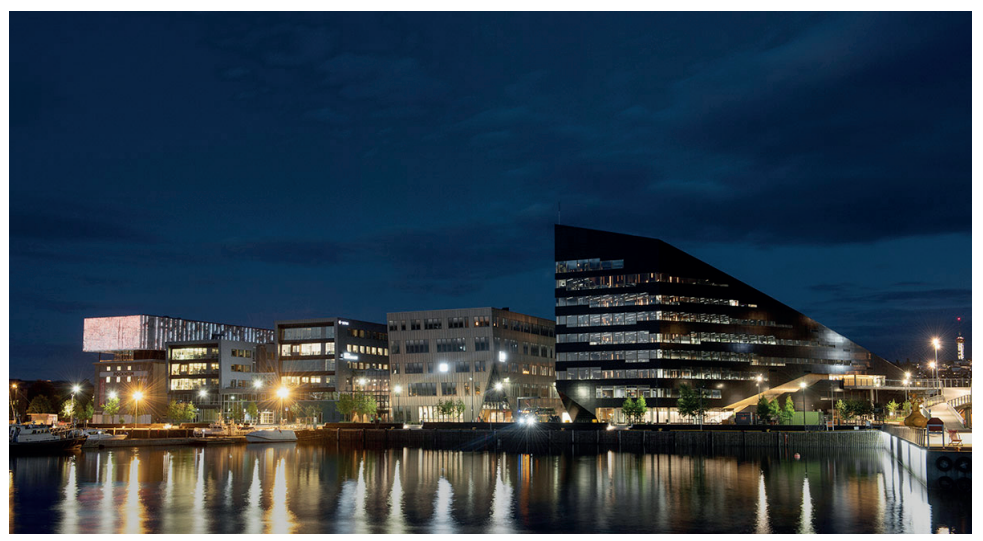


Este gran esfuerzo en la reorganización de nuestras ciudades debe estar acompañado por una mayor conciencia en las decisiones que se toman al proyectar cada infraestructura, priorizando en ellas la energía incorporada de los materiales a proyectar.

En ese sentido, es importante que se pueda realizar una cuantificación de manera general y luego a mayor detalle, según la etapa de cada proyecto y en relación con sus trámites municipales, e incluso aquellos para obtener la licencia de funcionamiento. Con ello se podría generar información necesaria para conocer cómo venimos actuando y tomar las medidas correspondientes, buscando la reducción y eficiencia de su consumo. Esta búsqueda deberá estar ligada a beneficios o mayores facilidades tributarias para todos los agentes involucrados en el proceso, como fabricantes de materiales, diseñadores, propietarios, inversionistas y cualquier otro que pueda ayudar en esta cadena.

De igual manera, es imperioso contar con una guía y certificaciones adaptadas a nuestras distintas realidades, las cuales puedan ser subvencionadas por el Estado, debido a que las actuales son certificaciones internacionales que tienen un costo y a las cuales no todos pueden acceder, lo que limita el acceso a la información y asesoría.

Las acciones descritas contribuirían al uso más eficiente de los materiales de construcción, lo que impactaría significativamente en el consumo del cemento, que tanto en el Perú como en el mundo constituye uno de los materiales más requeridos.

El cemento, con una producción de 4000 millones de toneladas al año, es responsable del $8 \%$ del $\mathrm{CO}_{2}$ generado en el mundo (Block, 2019), pues libera químicos reactivos al ambiente en el proceso de su fabricación. Posee cualidades que han favorecido su uso y expansión al ser un material fácil de utilizar, de bajo mantenimiento y por su larga vida útil. Esto permite que la alta energía empleada en su implementación se disipe en el tiempo. El problema principal es que no se cuenta con tiempo (décadas o siglos) para disgregar los impactos nocivos que pueda tener, más aún si se considera el impacto que significará la gran cantidad de metros cuadrados que se prevén construir hasta el 2060.

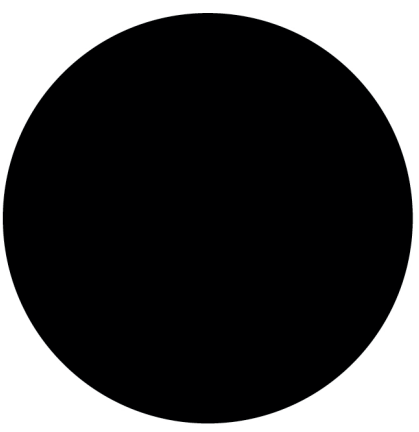

CABELLO HUMANO $60 \mathrm{um}$

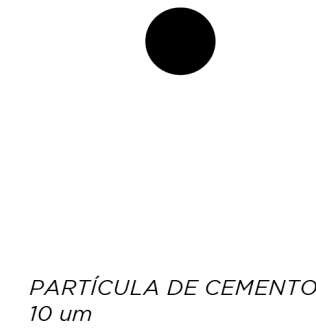

10 um
Figura 7.

Tamaño

de partícula

de cemento

Portland

Elaboración propia 
Conociendo la relevancia e impacto del mercado del cemento en el mundo y en nuestras ciudades, existen diversas investigaciones que buscan solucionar el problema de la contaminación que genera al producirlo, con el fin de que sea libre de emisiones.

En el año 2019, investigaciones a cargo del profesor Yet-Ming Chiang en el MIT intentaron capturar el dióxido de carbono generado en dos fases, antes de que este ingrese al medio ambiente. Sabina (2019) las detalla: la primera fase de liberación de carbono se produce por el calor necesario para su fabricación, el cual se podría disminuir haciendo uso de distintas fuentes de calor renovables, pero esto implica un gasto económico en su implementación. La segunda fase se da en los gases liberados en su reacción química; en este proceso se está enfocando el equipo de Chiang para convertir el carbonato de calcio de la piedra en hidróxido de calcio.

Por otro lado, en diversas partes del mundo existen otros esfuerzos que buscan cambiar los elementos que interactúan con el cemento al momento de la composición del concreto. Como señala Block (2018), un grupo de investigadores del Imperial College London apunta a generar soluciones al principal aglutinante del concreto, la arena, reconociendo que la extracción de este recurso a un ritmo muy acelerado afecta a países como la India, en donde se producen saqueos de este insumo que dañan los ecosistemas naturales. Por ese motivo, plantean el uso de arena del desierto como material de construcción biodegradable para la producción de bloques que posean la fortaleza del concreto, pero utilizando en su proceso la mitad de la huella de carbono de ese material. Además, podrían ser reciclados y reutilizados una vez acabada su finalidad, o pueden descomponerse naturalmente.

Por otro lado, Sabina (2018) describe la investigación realizada por la Universidad de Lancaster, Reino Unido, dirigida por el investigador principal Mohamed Saafi ${ }^{6}$, en asociación con la empresa de materiales sostenibles CelluComp ${ }^{7}$, que busca fortalecer las mezclas de concreto agregando nanopartículas extraídas de las zanahorias y hortalizas de raíz, lo que mejora las propiedades mecánicas y de microestructura, y utiliza menor cantidad de cemento. Esto ayudaría a reducir su consumo y, por ende, las emisiones asociadas a su fabricación.

En nuestro país, una investigación realizada por Arístides Sotomayor y George Power, publicada con el título de Tecnologías limpias y medio ambiente en el sector industrial peruano (2019), analiza el caso de dos empresas que buscan disminuir la producción de $\mathrm{CO}_{2}$ al medio ambiente: Cementos Inka y la Unión de Cementos Andinos (UNACEM). Dichos consorcios se enfocan en aplicar

6 Mohamed Saafi es profesor e investigador de la Universidad de Lancaster. Centra sus estudios en el desarrollo de materiales funcionales y sensores inteligentes para energía e infraestructura civil.

7 CelluComp es una empresa con sede en Escocia. Trabaja en el desarrollo y comercialización de materiales sostenibles. 
diferentes acciones dentro del proceso de fabricación del cemento, como la mejora de la tecnología de sus equipos, mejora de sus procesos de producción y control de las emisiones de partículas; del mismo modo, emplean diferentes fuentes de energía para su consumo reduciendo el uso de combustibles fósiles.

Estos esfuerzos deben reproducirse en el marco de un cambio en nuestras prioridades, para que estas excepciones se conviertan en una regularidad en la industria de la construcción.

\section{REDEFINIR PRIORIDADES}

Ayudar a nuestras ciudades y a nuestro planeta requiere de compromisos, de planificación, de hojas de ruta, de acciones, de activismo, de redefinir nuestras prioridades frente a la ciudad. Existen referencias importantes de distintas declaraciones contra el cambio climático que tienen como eje puntual la creación de conciencia en todas las personas involucradas en la cadena de infraestructuras, que va desde la fabricación de los materiales hasta la implementación, tratamiento de desechos y relación con los clientes.

La problemática suscitada por el crecimiento de las ciudades y el uso de materiales nocivos al ecosistema nos plantea cuestionamientos a partir de los diversos roles que asumimos. Al respecto, Harper (2019a) se pregunta “icómo sería una respuesta arquitectónica más radical a una era de emergencia climática? ¿Qué significaría para los diseñadores, constructores, comisionados y usuarios de edificios enfrentar una emergencia con ambiciones holísticas culturales y económicas, así como técnicas?” (párr. 6).

Desde un rol activista es necesario que más profesionales de distintas ramas sean actores de transformación para promover mayor rapidez en el proceso de cambio para la utilización y disponibilidad de materiales con baja huella de carbono. Desde nuestro rol como proyectistas y generadores de estrategias para el crecimiento y ordenamiento de las ciudades, debemos fomentar los lineamientos que apunten a repensar nuestras formas de proyectar infraestructuras, de baja y larga escala, en donde exista una sinergia clara entre nuestro medio ambiente, viabilidad económica, rentabilidad, ecología y ciudad. Debemos ser capaces de construir ejemplos claros donde estos criterios funcionen. Como señala Harper (2019b), en torno a la Trienal de Arquitectura en Oslo en el 2019, el desafío más existencial, emocionante y revolucionario que enfrenta la sociedad es rediseñar nuestra economía para proteger la única biosfera que tenemos (párr. 16). Además de la siguiente interrogante: ¿qué tipo de arquitectura crearemos cuando los edificios ya no sean instrumentos de acumulación financiera? (párr. 20).

De igual manera, es importante resaltar que se requiere de voces comprometidas y activas desde todos los frentes, como menciona Darran Anderson ${ }^{8}$ (2019).

8 Darran Anderson es un escritor irlandés, autor del libro Imaginary Cities (2015), elegido el libro del año por el Financial Times y The Guardian. 
Las pruebas que enfrentamos requieren el tipo de esfuerzos de infraestructuras colosales que se presenciaron en el New Deal, el Plan Marshall y el programa espacial, y hay más en juego. También requerirá la participación en campos más allá de los límites de cada disciplina. Cada enfoque singular es insuficiente por sí solo, dado que todos son tan interdependientes como lo es lo urbano con lo rural. Todavía son posibles otros futuros, siempre que podamos ver más allá de nuestros límites (párrs. 27-28).

Las respuestas también deben venir desde un ámbito político promoviendo reconocimientos a la arquitectura y urbanismo que considere la energía incorporada en sus propuestas. Si ya existen seis campos y veintidós temas en las bienales nacionales de arquitectura en el Perú, ¿no es posible considerar la sostenibilidad y la búsqueda de reducción del $\mathrm{CO}_{2}$ como uno nuevo en ellos? En los últimos años, arquitectos de diversas partes del mundo están tomando un rol más activista generando en conjunto acuerdos y declaraciones frente al cambio climático, como en el Reino Unido donde la UK Architects Declare Climate and Biodiversity Emergency (2019) ${ }^{9}$ señala lo siguiente:

\begin{abstract}
Las crisis gemelas del colapso climático y la pérdida de biodiversidad son el problema más grave de nuestro tiempo [...] Para todos los que trabajan en la industria de la construcción, satisfacer las necesidades de nuestra sociedad sin violar los límites ecológicos de la tierra exigirá un cambio de paradigma en nuestro comportamiento [...] La investigación y la tecnología existen para que podamos comenzar esa transformación ahora, pero lo que ha faltado es la voluntad colectiva. (párrs. 1- 3)
\end{abstract}

De igual manera, diferentes agrupaciones de arquitectos en veinticuatro países se han sumado a la lucha frente al cambio climático realizando en cada país una declaración de emergencia sobre el clima y la biodiversidad. En nuestro país el tema se diluye y pierde la relevancia que debería tener a pesar del impacto que tendrá. Esto debido a que el Perú se ha enfocado en otra serie de problemas importantes para la ciudad, como la reforma del transporte público, generación de espacios públicos de calidad, falta de infraestructura ambiental adecuada, de planeamiento y ejecución de los planes para las ciudades; sin embargo, advertidos y conscientes de la urgencia de su atención, debemos, desde cada uno de nuestros espacios, generar las acciones para su inclusión en las prioridades de estos tiempos.

9 UK Architects Declare Climate and Biodiversity Emergency es una declaración de la red de Architects Declare que involucra a un conjunto de oficinas de arquitectura del Reino Unido que aborda la emergencia climática y la biodiversidad. 
Figura 8

Declaración de Emergencia del Clima y de la

Biodiversidad de Arquitectos del Reino Unido

Fuente: Architects Declare (2019)

\section{CONCLUSIONES}

La disciplina de la arquitectura y el urbanismo siempre se encuentra en constante cambio y evolución, y hoy es más relevante que nunca en la historia incorporar como criterio indisociable el cambio climático en ellas. Las ciudades deben ser adaptadas para las implicancias que el cambio climático tendrá en ellas y este fenómeno deberá ser un factor determinante que condicionará cada vez más su diseño, crecimiento y composición, sobre todo en las urbes menos preparadas, como las del Perú.

El impacto provocado por el COVID-19, que ha develado aún más las desigualdades y diferencias urbanas que existen en nuestras ciudades, nos desnuda frente a una realidad que claramente indica que no estamos preparados; saberlo puede ser una gran ventaja.

El filósofo Bruno Latour ${ }^{10}$ (2020) escribió una reflexión al respecto, en la que relaciona esta crisis sanitaria, la del COVID-19, con el cambio climático. En dicho texto señaló lo siguiente: "Propongo la hipótesis, como muchas otras, de que la crisis de salud prepara, induce, incita a prepararnos para el cambio climático. Esta hipótesis aún necesita ser probada" (párr. 1).

10 Burno Latour es filósofo, antropólogo y sociólogo francés, que ha escrito más de veinte libros y publicado más de ciento cincuenta artículos. 
Los grandes cambios dentro de las ciudades, a través de la historia, se dieron siempre en situaciones extremas que nos llevaron al límite, solo que esta vez el límite está en camino, pero no nos estamos deteniendo a observarlo lo suficiente y nuestra reacción debería de ser aún más contundente. Como lo señala la joven activista ambiental Greta Thunberg ${ }^{11}$ (2019): "Quiero que actúes como lo harías en una crisis. Quiero que actúes como si nuestra casa estuviera en llamas, porque lo está" (párr. 18).

Por tal motivo, es importante acelerar la implementación de estas tres acciones prioritarias para reducir la producción de $\mathrm{CO}_{2}$ en nuestras ciudades; será prioridad la energía incorporada por ser transversal en ellas. Es necesario tener en cuenta que no solo existe un camino o un tipo de arquitectura o de planteamiento urbano para solucionar el tema, sino que deben plantearse todas las alternativas posibles, tomar consensos y actuar en consecuencia, para que nuevas formas de hacer arquitectura se vean reflejadas bajo este concepto.

Finalmente, existe un paso previo para conseguir que las acciones a implementarse puedan ser sostenibles en el tiempo y ello implica realizar el análisis estratégico de la data histórica y actual; sin embargo, nuestras ciudades carecen de ello. Por esa razón, es fundamental potenciar un sistema integrado de información catastral en todas las ciudades, como paso fundamental que permitirá tener la documentación necesaria para poder estudiar, analizar, evaluar y hacer seguimiento del uso de las edificaciones, la densificación y la energía incorporada de los nuevos proyectos en la ciudad. Con ello es posible determinar cada cierto periodo dónde, cuándo y cómo deberían seguir creciendo nuestras ciudades y sostener su desarrollo. Esta es quizá la manera más idónea de realmente proponer nuevos tipos de ciudades que reinventen y potencien las que tenemos, en prevención de lo que acontecerá en los próximos años.

\section{REFERENCIAS}

Anderson, D. (23 de enero del 2019). As Environmental Catastrophe Unfolds, We Need Architecture that is More than Just Green. Dezeen. https://www. dezeen.com/2019/01/23/darran-anderson-opinion-modular-adaptivearchitecture-environment-anthropocene/

Architects Declare. (30 de mayo del 2019). UK Architects Declare Climate and Biodiversity Emergency. https://www.architectsdeclare.com/

Block, I. (24 de marzo del 2018). New Material Made from Desert Sand Could Offer Low-Carbon Alternative to Concrete. Dezeen. https://www.dezeen. com/2018/03/24/desert-sand-could-offer-low-carbon-concrete-alternative/

Block, I. (20 de septiembre del 2019). Architects Should Give Up Concrete Say Experts at Architecture of Emergency Climate summit. Dezeen. https://www.dezeen. com/2019/09/20/concrete-climate-change-architecture-emergency/

11 Greta Thunberg es una activista ambiental, reconocida como personaje del año 2019 por la revista Time. 
Chakrabarti, V. (2013). A Country of Cities: A Manifesto for an Urban America. Metropolis Books.

Construcción y Vivienda. (2 de agosto del 2020). Plan de Desarrollo Urbano Lima 20212040 identifica más de 40 centralidades urbanas en proceso de formación. Periódico Construcción y Vivienda. https://www.construccionyvivienda. com/2020/08/02/plan-de-desarrollo-urbano-lima-2021-2040-identificamas-de-40-centralidades-urbanas-en-proceso-de-formacion/

Fairs, M. (17 de octubre del 2018). Dutch Design Week Anthropocene Design Opinion. Dezeen. https://www.dezeen.com/2018/10/17/dutch-design-week-anthro pocene-design-opinion/

Friedlingstein et al. (2020). The Global Carbon Budget 2020, Earth System Science Data. Global Carbon Atlas. http://globalcarbonatlas.org/en/CO2-emissions

Giles, C., y Song, W. (12 de octubre del 2020). US election 2020: What is Trump's record on the environment? BBC News. https://www.bbc.com/news/ election-us-2020-54103861

Harper, P. (11 de junio del 2019a). We're Seeing an Unprecedented Mobilisation of Architects in the Fight Against Climate Change. Dezeen. https:// www.dezeen.com/2019/06/11/radical-architecture-climate-changeopinion-phineas-harper/

Harper, P. (25 de septiembre del 2019b). Our Dependency on Growth, Like on Concrete, Must be Abolished. Dezeen. https://www.dezeen.com/2019/09/25/ oslo-architecture-triennale-architecture-degrowth-phineas-harper/?li_ source=LI\&li_medium=bottom_block_1

Kvaal, I. (2019). Powerhouse Brattørkaia, por Snøhetta Architects [Fotografía]. Arquine. https://www.arquine.com/powerhouse-brattorkaia/

Latour, B. (26 de marzo del 2020). Is This a Dress Rehearsal? Critical Inquiry. https:// critinq.wordpress.com/2020/03/26/is-this-a-dress-rehearsal/

Lezama, C. (4 de mayo del 2017). Vistas aéreas de Lima, centro financiero, construcciones, edificios, viviendas [Fotografía]. Andina. Agencia Peruana de Noticias. https://andina.pe/ingles/noticia-peru-limadistricts-withhighestdemandfor-online-housingsearch-665568.aspx

Sabina, R. (9 de agosto del 2018). Carrots Could be Key to Stronger Concrete. Dezeen. https://www.dezeen.com/2018/08/09/carrots-concrete-stronger-lan caster-university-technologyl

Sabina, R. (7 de octubre del 2019). MIT Researchers Develop Emissions-Free Cement Production Process. Dezeen. https://www.dezeen.com/2019/10/07/mitresearchers-emissions-free-cement/

Sotomayor-Cabrera, A., y Power-Porto, G. (2019). Tecnologías limpias y medio ambiente en el sector industrial peruano. Casos prácticos Perú. Universidad de Lima, Fondo Editorial. 
Thunberg, G. (25 de enero del 2019). Our House is on Fire. The Guardian. https://www. theguardian.com/environment/2019/jan/25/our-house-is-on-fire-gretathunberg16-urges-leaders-to-act-on-climate

Zen Stitching. (8 de mayo del 2020). What Is Boro Stitching. https://zenstitching.ca/ blog/2020/5/8/what-is-boro-stitching 